\title{
Analytic MHD theory for Earth's bow shock at low Mach numbers
}

\author{
Crockett L. Grabbe and Iver H. Cairns \\ Department of Physics and Astronomy, University of Iowa, Iowa City
}

NASA-CR-202734

\begin{abstract}
A previous MHD theory for the density jump at the Earth's bow shock, which assumed the Alfven $\left(M_{A}\right)$ and sonic $\left(M_{s}\right)$ Mach numbers are both $\gg 1$, is reanalyzed and generalized. It is shown that the MHD jump equation can be analytically solved much more directly using perturbation theory, with the ordering determined by $M_{A}$ and $M_{s}$, and that the first-order perturbation solution is identical to the solution found in the earlier theory. The second-order perturbation solution is calculated, whereas the earlier approach cannot be used to obtain it. The second-order terms generally are important over most of the range of $M_{A}$ and $M_{s}$ in the solar wind when the angle $\theta$ between the normal to the bow shock and magnetic field is not close to $0^{\circ}$ or $180^{\circ}$ (the solutions are symmetric about $90^{\circ}$ ). This new perturbation solution is generally accurate under most solar wind conditions at 1 $\mathrm{AU}$, with the exception of low Mach numbers when $\theta$ is close to $90^{\circ}$. In this exceptional case the new solution does not improve on the first-order solutions obtained earlier, and the predicted density ratio can vary by $10-20 \%$ from the exact numerical MHD solutions. For $\theta \sim 90^{\circ}$ another perturbation solution is derived that predicts the density ratio much more accurately. This second solution is typically accurate for quasi-perpendicular conditions. Taken together, these two analytical solutions are generally accurate for the Earth's bow shock, except in the rare circumstance that $M_{A} \leq 2$. MHD and gasdynamic simulations have produced empirical models in which the shock's standoff distance $a_{s}$ is linearly related to the density jump ratio $\mathrm{X}$ at the subsolar point. Using an empirical relationship between $a_{s}$ and $X$ obtained from MHD simulations, $a_{s}$ values predicted using the MHD solutions for $X$ are compared with the predictions of phenomenological models commonly used for modeling observational data, and with the predictions of a modified phenomenological model proposed recently. The similarities and differences between these results are illustrated using plots of $\mathrm{X}$ and $a_{s}$ predicted for the Earth's bow shock. The plots show that the new analytic solutions agree very well with the exact numerical MHD solutions and that these MHD solutions should replace the the corresponding phenomenological relations in comparisons with data. Furthermore, significant differences exist between the standoff distances predicted at low $M_{A}$ using the MHD models versus those predicted by the new modified phenomenological model. These differences should be amenable to observational testing.
\end{abstract}

\section{Introduction}

Research on the position of the Earth's bow shock, which has been pursued for the last 3 decades, has predominately focused on gasdynamic and quasi-gasdynamic analysis [e.g., Spreiter et al., 1966; Fairfield, 1971; Formisano et al., 1971; Holzer and Slavin, 1978; Slavin et al., 1983; Farris et al., 1991]. There are strong motivations, however, for further developing an MHD theory of the shock's location [Cairns and Grabbe, 1994], since the bow shock is a magnetosonic shock wave and the solar wind interacting with the Earth's magnetosphere is a magnetized plasma. Robust MHD theories

Copyright 1995 by the American Geophysical Union.

Paper number 95JA01286.

0148-0227/95/95JA-01286\$05.00 show dependence on the Alfven Mach number $M_{A}$, the sonic Mach number $M_{3}$, and the angle $\theta$ between the magnetic field and shock normal vectors. In contrast, gasdynamic theory is a function of the lone Mach number $M_{s}$ (phenomenological substitutions of $M_{A}$ or $M_{m}$, for $M_{s}$ are often made to model the Earth bow shock in variants on gasdynamic theory) and its validity is generally limited to $M_{A}^{2} \gg M_{s}^{2} \gg 1$. (Here $M_{m s}$ is the $\theta$-dependent magnetosonic Mach number.) Our focus is on developing an accurate model of the solar wind interaction for low $M_{A}$ and $M_{s}$, which requires an MHD analysis.

An earlier effort at a theoretical analysis of the bow shock using MHD theory was pursued by Zhuang and Russell [1981]. They showed that the jump conditions lead to a complicated cubic equation for the density jump ratio as a function of the magnetic field compo- 
nents and temperature in the solar wind. Their paper presents an involved method for solving that equation for large $M_{A}$ and $M_{3}$ in which the nonlinear algebraic equation for the density jump ratio $X=\rho_{s w} / \rho_{b}$ s is converted into 30 linear equations $\left(\rho_{s w}\right.$ and $\rho_{b s}$ are the mass density of the upstream solar wind and immediately downstream of the bow shock, respectively). These equations were solved to obtain analytical solutions to first order in the Mach numbers, both of which were assumed to be $>>1$.

There is interest in the density jump ratio $X$ for two reasons. One is intrinsic, since spacecraft experiments measure $X$ directly at the bow shock. The other is its relationship to the standoff distance of the bow shock. Empirical analysis has shown the magnetosheath thickness (the bow shock standoff distance less the known distance to the magnetopause) is proportional to $X$, so knowing $X$ allows predictions for the standoff distance [Spreiter et al., 1966; Cairns and Grabbe, 1994; Cairns and Lyon, 1995]. The difficulty with using the ZhuangRussell analytical solution for comparison with data on the bow shock is that when $M_{A}$ or $M_{2}$ is sufficiently below 10, their solution generally underestimates $X$. Our analysis indicates that the underestimation can be significant for all $\theta$ except for $\theta$ relatively close to 0 . This inaccuracy degrades the usefulness of the theory in modeling most shocks which are observed at these lower Mach numbers.

Historically the trend in investigating the bow shock position has been to utilize gasdynamic results for comparison with the observed shock standoff distances, although efforts were made to introduce some MHD concepts into the gasdynamic model. Spreiter and Rizzi [1974] present MHD solutions for solar wind magnetic fields aligned with the flow $(\theta=0)$, in which case the MHD equations reduce to a set of equations directly analogous to the gasdynamic equations except for a modified equation of state. Spreiter and Stahara [1980] introduced a global quasi-gasdynamic model oriented toward its usability for comparison with data in which the flow is determined by solving the conservation equations with the magnetic field terms neglected, and the magnetic field is then determined from the gasdynamic flow using the magnetic induction equation.

Russell [1985] argued how the difficulty of comparing with MHD theory made the quasi-gasdynamic model more usable for data comparison and that the single Mach number in the quasi-gasdynamic theory (which is the sonic Mach number $M_{s}$ in the gasdynamic equations) should be replaced with the magnetosonic Mach number $M_{m s}$, as was done previously by Formisano et al. [1971] when modeling shock locations. This phenomenological model, in which $M_{m s}$ is substituted for $M_{2}$ in the gasdynamic equation obtained for $a_{s}$ by Spreiter et al. [1966], has often been used to analyze spacecraft data for the Earth bow shock [e.g. Farris et al., 1991]. After comparing some observed bow shock crossings with gasdynamic theory, Slavin et al. [1983] state that there appears to be no theoretical support for this substitution, and that the arguments advanced for the substitution appear valid only under conditions of strong IMF intensity or low $M_{A}$. Cairns and Grabbe [1994] demonstrate the lack of theoretical foundation for this substitution. Russell and Zhang [1992] and Cairns et al. [1995] have shown that this phenomenological model is observationally inadequate at low $M_{A}$ and $M_{m s} \leq 3$, thereby indicating a need for further developing the theory at these low Mach numbers. Recently, Farris and Russell [1994] proposed a modified phenomenological model for $a_{s}$ for low $M_{A}$.

These developments and the continued reliance on phenomenological models for data comparison clearly indicate a need for further developing MHD theory especially at low Mach numbers. Cairns and Grabbe [1994] considered the obvious MHD generalization of Spreiter et al.'s [1966] gasdynamic theory for $a_{\mathrm{a}}$ : linearly relating the MHD density jump ratio $X$ (rather than the gasdynamic $X$ ) to the magnetosheath thickness. They then showed that the resulting theory for $a_{s}$ reduces to the phenomenological $M_{m}$. model only under restricted circumstances which are generally not met in the solar wind. Their theory shows direct dependences on $M_{A}, M_{s}, \theta$, and specific heat ratio ? that cannot be subsumed into $M_{m}$, alone, and reduces to the gasdynamic theory when $M_{A}>M_{1}>1$ Subsequently, Cairns and Lyon [1995] presented threedimensional ideal MHD simulations for $\theta \geq 45^{\circ}$, which show that the magnetosheath thickness is indeed proportional to the MHD value of $\mathrm{X}$ for $M_{A} \geq 1.5$. This was utilized to extend the MHD model for $a$, developed by Cairns and Grabbe [1994].

The present paper markedly extends the Cairns ana Grabbe [1994] MHD analysis by obtaining analytical solutions of $X$ for all $\theta$, linking them with the MHD simulations of Cairns and Lyon [1995], and comparing the predictions with the modified phenomenological model of Farris and Russell [1994]. In section 2 it will be shown that the MHD jump conditions can be analytically solved, in a much more direct fashion than was done earlier, by using perturbation theory. The solution given by Zhuang and Russell [1981] is the same as the first-order perturbative solution which is obtained much more directly. Furthermore, with perturbation theory the second-order (and even higher) terms in the solution are obtained, whereas they cannot be in the approach used by Zhuang and Russell. Two solutions accurate to second order are obtained for separate overlapping ranges of $\theta$ : approximately $0^{\circ}$ to $75^{\circ}$ and $50^{\circ}$ to $90^{\circ}$ (with symmetry about $90^{\circ}$ ). The second- and higher-order terms are determined to be important for $\theta$ not close to $0^{\circ}$ except when both $M_{A}$ and $M_{8}$ are $>>1$. Taken together, these two analytical solutions are generally accurate to within a few percent under most conditions that exist in the solar wind at $1 \mathrm{AU}$. Section 3 compares the low-Mach number predictions from both the new analytic theory, and from magne- 
tosonic phenomenological models that have been used in spacecraft data analysis, against the precise MHD results. These comparisons are made between the shock standoff distances predicted by selected empirical models. Section 4 summarizes the major conclusions of this paper.

\section{Density and Velocity Jump}

The Rankine-Hugoniot relations for the conservation of mass, energy, momentum, and charge density, along with Maxwell's equations, constrain the changes in fluid and electromagnetic variables across the shock. Zhuang and Russell [1981] used these relations to derive a cubic equation for the upstream to downstream density ratio $X=\rho_{s w} / \rho_{b s}=U_{b s} / U_{s w}$ (Bw denotes the solar wind upstream and bs the downstream side of the bow shock, while the symbols $U$ denote fluid speeds). This equation has some differences from a similar cubic derived for shock waves using the Vlasov (rather than a fluid) approach of Tidman and Krall [1971]. The Vlasov derivation assumed Maxwellian electron and ion distributions, which are probably not valid near the Earth's bow shock. The fluid equation can be expressed in the following streamlined form [see Cairns and Grabbe, 1994]:

$$
X^{3}+C_{a} X^{2}+C_{b} X+C_{c}=0
$$

where the coefficients are

$$
\begin{aligned}
C_{a}= & -\left\{\frac{(\gamma-1)}{\gamma+1)}+\frac{\gamma+(\gamma+2) \cos ^{2} \theta}{(\gamma+1) M_{A}^{2}}\right. \\
& \left.+\frac{2}{(\gamma+1) M_{s}^{2}}\right\} \\
C_{b}= & \frac{1}{(\gamma+1) M_{A}^{2}}\left\{\gamma\left(1+\cos ^{2} \theta\right)-2\right. \\
& \left.+\cos ^{2} \theta\left[\frac{(\gamma+1)}{\left(M_{A}^{2}\right.}+\frac{4}{M_{s}^{2}}\right]\right\} \\
C_{c}= & -\frac{\cos ^{2} \theta}{\left.(\gamma+1) M_{A}^{4}\right)}\left[(\gamma-1)+\frac{2 \cos ^{2} \theta}{M_{s}^{2}}\right]
\end{aligned}
$$

where $M_{A}=U_{s w} / U_{A}$ and $M_{s}=U_{s w} / c_{s}$ are the Alfven and sonic Mach numbers, $\theta$ is the angle between the shock normal and the solar wind magnetic field $\mathbf{B}$, and $\gamma$ is the adiabatic constant. Here $U_{s w}$ is the solar wind speed, $U_{A}$ is the Alfven speed, and $c_{s}$ the ion acoustic speed. The analysis by Cairns and Grabbe [1994] will be extended by finding analytical solutions over the entire range of $\theta$.

While (1) has three solutions for any set of plasma parameters, only one corresponds to a solution for the Earth's bow shock. For $\theta$ close to $0^{\circ}$, the other two solutions correspond to "switch-on" shocks $\left(X \sim 1 / M_{A}^{2}\right)$, while for $\theta$ in the vicinity of $90^{\circ}$ the other two solutions represent an unphysical "negative-density" shock (mass density of opposite sign on the two sides of the shock) and an unphysical "reverse" shock $(X<0.25)$. Zhuang and Russell [1981] used the cubic formula to analyti- cally solve (1) for the bow shock under the restriction that $M_{A}$ and $M_{2} \gg 1$. Unfortunately, that approach ties the solution up into a Gordian knot. To unravel this knot, they broke the cubic solution into 30 linear equations, which were solved simultaneously for large $M_{A}$ and $M_{\mathbf{A}}$.

A better approach for finding analytical solutions avoids using the cubic formula altogether. Since the two Mach numbers are both $>1$ for Earth's bow shock, a direct approach is to solve the equation using perturbation theory [e.g., Nayfeh, 1993], with the perturbation parameters being $1 / M_{A}^{2}$ and $1 / M_{a}^{2}$. These are two different perturbation parameters, but they will be taken as the same order $\epsilon$ in our analysis since they are approximately of the same order in the solar wind. We will denote the order given by these two parameters as $\epsilon$. Thus the ordered solution we are seeking takes the form:

$$
X=x_{0}+\epsilon x_{1}+\epsilon^{2} x_{2}+\ldots
$$

The coefficient terms in (1) are similarly ordered into powers of $\epsilon$ :

$$
\begin{aligned}
& C_{a}=-R_{a}-\epsilon S_{a} \\
& C_{b}=\epsilon S_{b}+\epsilon^{2} T_{b} \\
& C_{c}=-\epsilon^{2} T_{c}-\epsilon^{3} U_{c}
\end{aligned}
$$

where the new variables are

$$
\begin{aligned}
R_{a} & =\frac{(\gamma-1)}{(\gamma+1)} \\
S_{a} & =\frac{2}{(\gamma+1) M_{a}^{2}}+\frac{\gamma+(\gamma+2) \cos ^{2} \theta}{(\gamma+1) M_{A}^{2}} \\
S_{b} & =\frac{\gamma \cos ^{2} \theta+(\gamma-2)}{(\gamma+1) M_{A}^{2}} \\
T_{b} & =\frac{\cos ^{2} \theta}{(\gamma+1) M_{A}^{2}}\left[\frac{4}{M_{a}^{2}}+\frac{(\gamma+1)}{M_{A}^{2}}\right] \\
T_{c} & =\frac{(\gamma-1) \cos ^{2} \theta}{(\gamma+1) M_{A}^{4}} \\
U_{c} & =\frac{2 \cos ^{4} \theta}{(\gamma+1) M_{a}^{2} M_{A}^{4}}
\end{aligned}
$$

From these orderings the perturbation equations for $O\left(\epsilon^{n}\right)$ can be written in a straightforward manner. The $O\left(\epsilon^{0}\right)$ equation just gives an expression for $x_{0}$, which can then be substituted into the $O(\epsilon)$ equation to algebraically solve for $x_{1}$ :

$$
\begin{aligned}
& x_{0}=R_{a} \\
& x_{1}=S_{a}-S_{b} / R_{a}
\end{aligned}
$$

Combining $x_{0}$ and $x_{1}$ then gives a solution for $\mathrm{X}$ to first order in $\epsilon$ :

$$
X=\frac{(\gamma-1)}{(\gamma+1)}+\frac{2}{(\gamma+1)]}\left[\frac{1}{M_{s}^{2}}+\frac{\sin ^{2} \theta}{(\gamma-1) M_{A}^{2}}\right]
$$

This solution is precisely the same as that obtained by 
Zhuang and Russell [1981, equation 43] through their analysis with 30 linear equations.

In addition to the more direct route in obtaining the first-order solution, the advantage of the perturbation technique is that the solution can be extended to the next higher order in $\epsilon$ without difficulty. The secondorder equation is

$$
\begin{array}{r}
3 x_{0}\left(x_{1}^{2}+x_{0} x_{2}\right)-\left[R_{a}\left(x_{1}^{2}+2 x_{0} x_{2}\right)\right. \\
\left.+2 x_{0} x_{1} S_{a}\right]+\left(x_{0} T_{b}+x_{1} S_{b}\right)-T_{c}=0
\end{array}
$$

Solving this equation, the solution for $X$ to second order becomes

$$
\begin{aligned}
X= & \frac{(\gamma-1)}{(\gamma+1)}+\frac{2}{(\gamma+1)}\left[\frac{1}{M_{*}^{2}}+\frac{\sin ^{2} \theta}{(\gamma-1) M_{A}^{2}}\right] \\
& +\frac{2 \sin ^{2} \theta}{(\gamma-1)^{2} M_{A}^{2}}\left\{\frac{\gamma \cos ^{2} \theta}{(\gamma-1) M_{A}^{2}}+(\gamma-2)\left[\frac{1}{M_{\theta}^{2}}\right.\right. \\
& \left.\left.+\frac{1}{(\gamma-1) M_{A}^{2}}\right]\right\}
\end{aligned}
$$

Note that the second-order term in (19) disappears if $\theta=90^{\circ}$ and $\gamma$ is taken as 2. This characteristic behavior may have played a role in the conclusion that $\gamma=2$ which Zhuang and Russell obtained by comparing data with their first-order theoretical results. Their data set could well have contained a preponderance of quasiperpendicular shocks. Thus the limitations of their first-order theory may have created an inherent bias in the data comparison toward an extrapolated value for $\gamma$ which minimizes the second-order corrective term, that is, toward $\gamma=2$ for cases with $\theta \sim 90^{\circ}$. This was very close to the average value actually determined in their analysis. Later data analyses [e.g., Farris et al., 1991] have concluded that the value of $\gamma$ is actually closer to $5 / 3$.

In fact, when $\theta$ is close to $90^{\circ}$ the foregoing perturbation expansion becomes relatively inaccurate because the contribution of the second-order terms becomes small (the $\gamma-2$ terms become predominant), and thus third- and higher-order terms in the perturbation expansion become important except when both Mach numbers are $>1$. In this case, however, the solution can be replaced with a better one. In (1) the coefficient $C_{c}$ and two terms in $C_{b}$ become of third or higher order in a modified perturbation ordering because the $\cos ^{2} \theta$ factor becomes very small (going to 0 at $\theta=90^{\circ}$ ). By neglecting these third-or-higher order terms the equation for $\mathrm{X}$ becomes quadratic and is easily solved:

$$
\begin{aligned}
& X= \frac{-C_{a}}{2}\{1+ \\
&\left.\sqrt{1-4\left[\left(\gamma+\gamma \cos ^{2} \theta-2\right) /(\gamma+1) C_{a}^{2} M_{A}^{2}\right]}\right\}
\end{aligned}
$$

where $C_{a}$ is just the coefficient given by (2). This solution is accurate to second or higher order for $\theta$ away from $90^{\circ}$ (as far down as $50^{\circ}-60^{\circ}$ ), and collapses to the solution by Cairns and Grabbe [1994] as $\theta \rightarrow 90^{\circ}$.
Taken together, (19) and (20) constitute reasonably simple analytical relationships derived from MHD theory for the density jump across the bow shock. These expressions can be easily compared with data. Note that these two solutions have a clear dependence on four variables: $M_{A}, M_{3}, \theta$, and $\gamma$, as expected theoretically [Cairns and Grabbe, 1994]. This variable dependence is missing in previous theoretical models for the bow shock location that use expressions for the density jump based on gas dynamics. Such models have previously predominated comparisons with data on the Earth's bow shock location; the dependence on 4 variables is only present in the Zhuang-Russell MHD model and in our recent MHD work [Cairns and Grabbe, 1994; Cairns and Lyon, 1995; and this paper].

Predictions for $\mathrm{X}$ as a function of $M_{\mathcal{A}}$ are compared in Figures 1-5 for the analytical solution from either (19) or (20), the exact numerical MHD solution, and the first-order solution of Zhuang and Russell [1981]. It should be noted that Zhuang and Russell derived their results under the assumptions that $M_{A}$ and $M_{s}$ are $>>1$. Therefore curves of their results for the low Mach numbers are used here only for the purpose of comparison with the predictions of (19) and (20), whose validity definitely does extend to lower Mach numbers.

In Figures 1-3 the analytic solutions for $X$ as a function of $M_{A}$ are compared for four angles between the magnetic field direction and the shock normal $(\theta=$ $\left.0^{\circ}, 30^{\circ}, 60^{\circ}, 90^{\circ}\right)$ and for three different sonic Mach numbers $\left(M_{3}=8,4,2\right)$. Figures 1 and 2 span the range of $M_{s}$ generally observed in the solar wind at $1 \mathrm{AU}$ [e.g., Fairfield, 1971, Figure 3]. Figure 3 is for a value of $M$, just below the most extreme cases observed in the solar

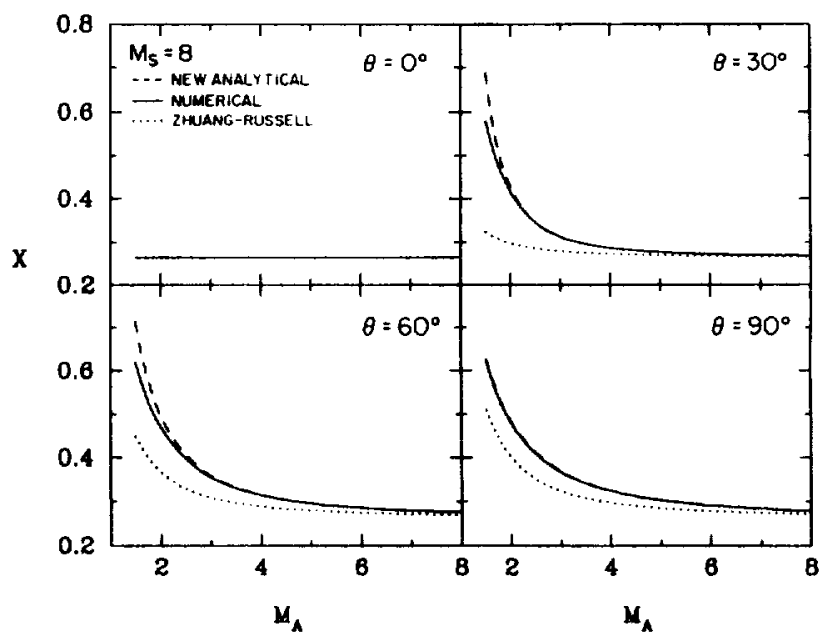

Figure 1. Comparison of the density ratio $X=$ $\rho_{s w} / \rho_{b}$, predicted by the second-order perturbation MHD solutions (19) and (20) (dashed lines) with the exact numerical MHD solution (solid lines) and the Zhuang-Russell solution (dotted lines), as a function of Alfven Mach number $M_{A}$ and selected $\theta$ (angle between the bow shock normal and the IMF) for sonic Mach number $M_{\mathrm{s}}=8$. The adiabatic constant $\gamma=5 / 3$ is used in all plots. 


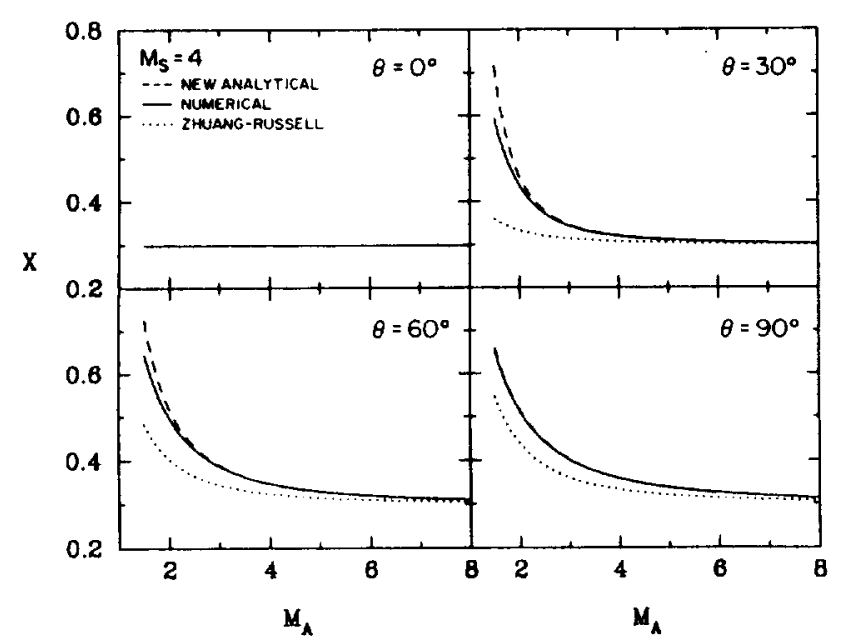

Figure 2. Same as Figure 1 for $M_{\mathrm{s}}=4$.

wind and is used to test how far the theory extends. All three figures show that the second-order solutions (19) and (20) predict the exact numerical results more accurately than the first-order Zhuang-Russell solution for all angles except for $\theta=0^{\circ}$, where both the new analytical solution and the Zhuang-Russell solution agree precisely with the exact numerical solution.

Figures 1-3 show that the Zhuang-Russell result for the low Mach-numbers becomes increasingly inaccurate as $M_{A}$ decreases for a given $\theta$, and that the their solution underestimates the exact solution for finite $\theta$. The Zhuang-Russell solution shows a noticeable error for all three nonzero angles in each of Figures 1-3. The analytic solutions contained in (19) and (20) show relatively small errors at intermediate $\theta\left(30^{\circ}\right.$ and $\left.60^{\circ}\right)$ when $M_{A}$ goes below 2-3.

Figures 4 and 5 show how these predictions for $X$ vary with $\theta$. In Figure 4 the value of $X$ given by (19) is plotted as a function of $\theta$ (ranging from $0^{\circ}$ to $70^{\circ}$ ) for $M_{s}=4$ and selected values of $M_{A}$. These plots show that the error in the analytic theory becomes significant at most angles when $M_{A}=2$, although they are gener-

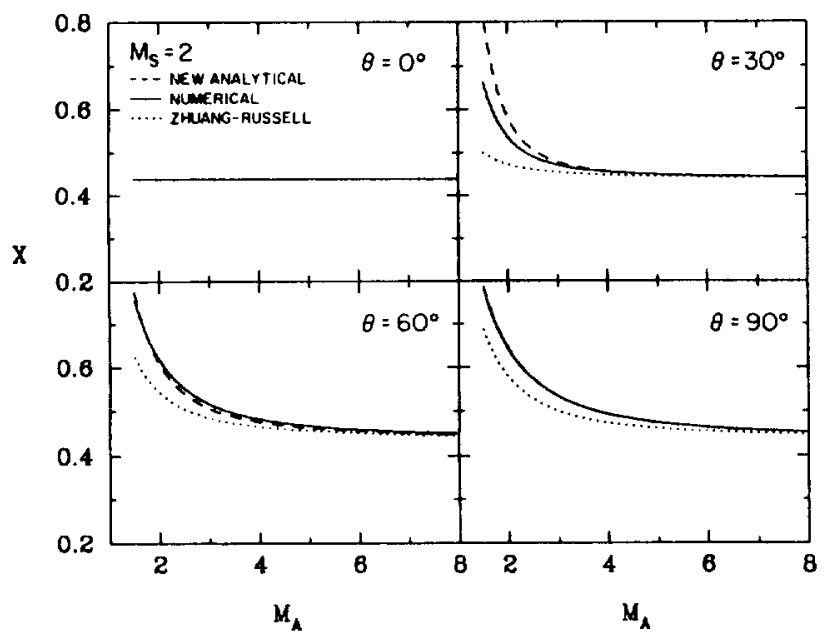

Figure 3. Same as Figure 1 for $M_{s}=2$.

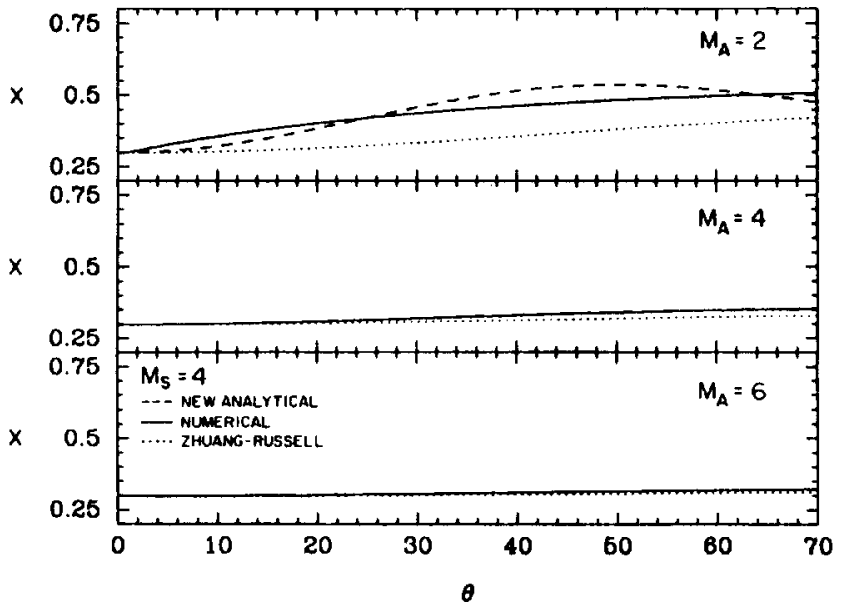

Figure 4. Comparison of the density ratio $X$ predicted by (19) with the exact numerical MHD solution and the Zhang-Russell solution, as a function of $\theta$ ranging $0^{\circ}-70^{\circ}$ for $M_{s}=4$ and selected small values of $M_{A}$.

ally negligible for higher $M_{A}$. In all cases (19) is more accurate the Zhuang-Russell result.

In Figure 5 the value of $\mathrm{X}$ given by (20) is plotted as a function of $\theta$ (ranging from $60^{\circ}$ to $90^{\circ}$ ) for $M_{s}=4$ and selected values of $M_{A}$. Figure 5 shows that (20) is very accurate (within about $1-2 \%$ ) for $M_{A} \geq 1.5$ for the approximate range of $\theta=75^{\circ}-90^{\circ}$ and only shows more significant error in the range $\theta=60^{\circ}-75^{\circ}$ for the case that $M_{A}=1.5$. In all cases the first-order result of Zhang and Russell is less accurate.

\section{Analytical Solutions and Phenomenological Models}

Equations (19) and (20), which were derived analytically from MHD theory, constitute useful analytical solutions that both complement the analysis by Cairns and Grabbe [1994] and can be easily compared with

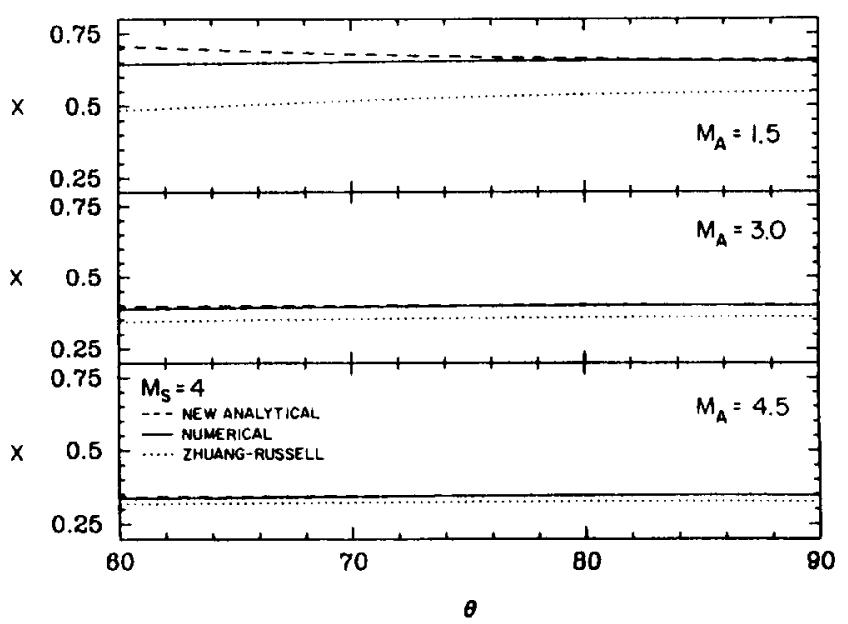

Figure 5. Comparison of the density ratio $X$ predicted by (20) with the exact numerical MHD solution and the Zhang-Russell solution, as a function of $\theta$ ranging $60^{\circ}-90^{\circ}$ for $M_{s}=4$ and selected small values of $M_{A}$. 
shock crossing data. As discussed in section 1 gasdynamic [Seiff, 1962; Spreiter et al., 1966] and MHD simulations for $\theta \geq 45^{\circ}$ [Cairns and Lyon, 1995] show empirically that the standoff distance $a_{\text {, }}$ is linearly dependent on the density jump $X$. (Note that $a_{s}$ is defined here as the distance between Earth's center and the shock's nose; in the aerodynamic literature "standoff distance" is defined as the distance of the obstacle to the shock, so an alternative would be to call $a$, the "geocentric shock distance.") This section examines and compares the predictions of models for $a_{s}$, that are constructed from MHD and gasdynamic solutions for $X$ combined with the empirical relationships between $a_{2}$ and $X$, for parameter ranges that can occur at the Earth's bow shock.

These empirical relationships between $a$, and $X=$ $\rho_{s w} / \rho_{b s}$ take the following form [Seiff, 1962; Spreiter et al., 1966; Farris and Russell, 1994;Cairns and Lyon, 1995]:

$$
\frac{a_{s}}{a_{m p}}=j+k X
$$

Here $a_{m p}$ is the distance from Earth to the nose of the magnetopause. It should be emphasized that although the analytical solutions found in section 2 for the density ratio $X$ apply locally everywhere on the bow shock, these empirical models are restricted to the subsolar region (the nose) of the shock. In the more general case, $a_{2}$ depends on (at least) five parameters: the four parameters $\left(M_{A}, M_{s}, \theta\right.$, and $\left.\gamma\right)$ that $X$ depends on, as well as the magnetopause shape [Spreiter and Stahara, 1980; Slavin et al., 1983].

For the gasdynamic empirical relation found by Seiff [1962] and further developed by Spreiter et al. [1966] $j=1$ and $k=1.1$, where the value of $k$ depends on the obstacle shape. This model has been the prevailing one used for published comparisons with spacecraft data for many years. Solving the gasdynamic equations for the density jump $X$ yields

$$
X_{g d}=\frac{(\gamma-1) M_{s}^{2}+2}{(\gamma+1) M_{s}^{2}}
$$

The phenomenological model of Formisano et al. [1971] and Farris et al. [1991] substituted $M_{m}$, for $M_{\text {. }}$. See Cairns and Grabbe [1994] for arguments against this procedure.

Two alternative empirical models have been developed recently, principally due to difficulties accounting for distant shock locations at low $M_{A}$ and $M_{m}$ s [Russell and Zhang, 1992; Cairns et al., 1995]. In the model presented by Farris and Russell [1994] the value for $k$ is modified from the Seiff-Spreiter form at lower Mach numbers by $k=1.1 M_{m s}^{2} /\left(M_{m s}^{2}-1\right)$ while $j$ stays 1. In the model developed from MHD simulations by Cairns and Lyon [1995], $j=0.4$ and $k=3.4$ for quasiperpendicular flows with $M_{3} \sim 8$ and $M_{A}>1.5$. This model explains their simulation results with excellent accuracy. Both the alternative models were developed with the goal of extending the relationship (21) to lower Mach numbers and exhibit a greater dependence on the density ratio at these low Mach numbers. It should be noted that for larger Mach numbers $(>5-10)$, $\rho_{s w} / \rho_{b}, \sim 0.25$ and all three empirical models are in approximate agreement.

A note regarding the importance of $\theta$ for these empirical models is in order. Cairns and Lyon [1995] point out that $a_{s}$ depends strongly on $\theta$, as evidenced by the qualitatively opposite variations of $a$, with decreasing

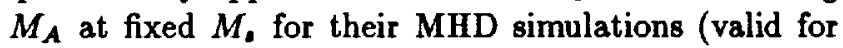
$\left.\theta \geq 45^{\circ}\right)$ compared to the field-aligned $\left(\theta=0^{\circ}\right)$ MHD simulations of Spreiter and Rizzi [1974]; that is, $a$, is predicted to increase with decreasing $M_{A}$ for $\theta \geq 45^{\circ}$ but to decrease with decreasing $M_{A}$ for $\theta=0^{\circ}$. The phenomenological models also predict variations in $a$, opposite to the Spreiter-Rizzi field-aligned MHD simulations, and this characteristic also argues against the validity of the phenomenological models near $\theta=0^{\circ}$. It is presently unknown how large $\theta$ must be for models based on (21) to hold, but the empirical models are likely relevant for $\theta \geq 20^{\circ}$. The recent observational results of Peredo et al. [1995], who find statistically that $a_{s}$ decreases with decreasing $M_{A}(>2)$ but do not specify $\theta$, make the dependence of $a$, on $\theta$ particularly topical.

In Figure 6 comparisons are made between the predictions for $a_{s} / a_{m p}$, based on the Cairns-Lyon empirical values of $j$ and $k$, for various $\theta\left(0^{\circ}, 30^{\circ}, 60^{\circ}, 90^{\circ}\right)$ at $M_{s}=8$. The solid line shows the exact numerical

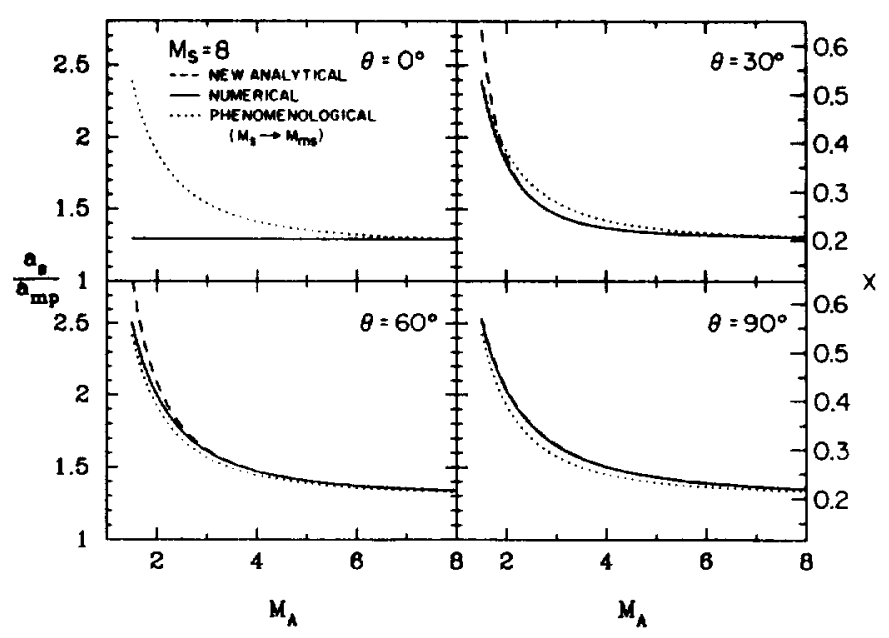

Figure 6. Comparison between the second-order analytical MHD solution (dashed lines), the numerical MHD solution (solid lines) and the gasdynamic solution with a phenomenological substitution of the magnetosonic Mach number $M_{m}$, for the sonic Mach number $M_{0}$ (dotted line). Here $\gamma=5 / 3$ and $M_{0}=8$. The axis on the left-hand side is $a_{s} / a_{\text {mp }}$ predicted for both models using the empirical relation between $X$ and $a_{s} / a_{m p}$ found in Cairns and Lyon's [1994] simulations. It is expected that this empirical relation is wrong for the $\theta=0^{\circ}$ case (from the Spreiter and Rizzi [1974] studies at $\theta=0^{\circ}$ ) so the value of $X$ from which the standoff distance is determined is shown on the right-hand side. 


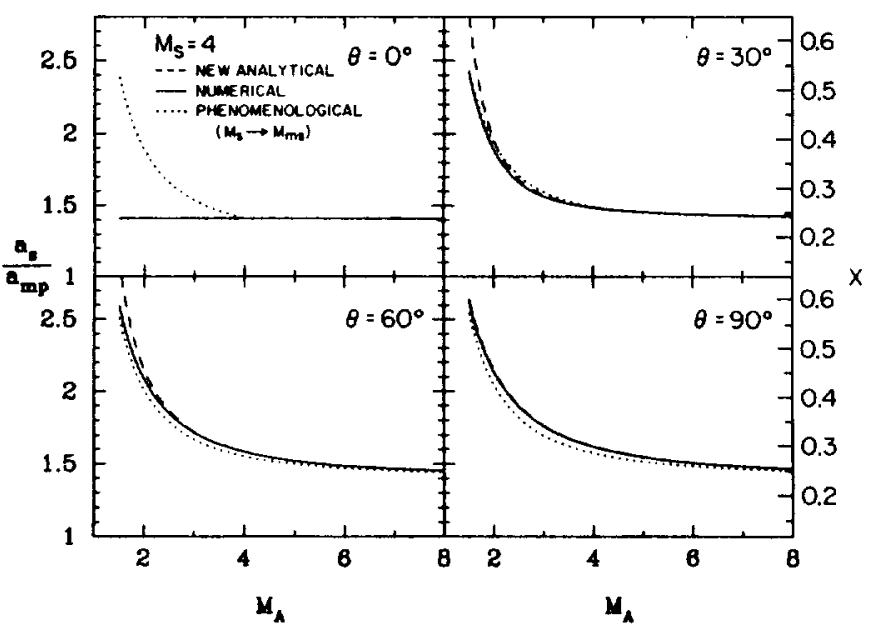

Figure 7. Same as Fig. 6 for the case that $M_{s}=4$.

MHD solutions, while the dashed line uses the analytical solutions (19) and (20) for $X$. The dotted line shows the prediction for the phenomenological $M_{m s}$ model of Formisano et al. [1971] and Farris and Russell [1991] (substitute $M_{m s}$ for $M_{s}$ in (22) and substitute the result into (21)) with the modification $j=0.4$ and $k=3.4$. Note that Cairns and Lyon [1995] show that, on the other hand, retaining the Seiff-Spreiter relationship $j=1$ and $k=1.1$ yields predictions for the phenomenological model that are widely different from their $M_{A} \leq 5$ simulation results. Since the empirical models are not valid for small $\theta$, that is, $\leqslant 20^{\circ}$ (see previous paragraph), a scale corresponding to $X$ is given on the right-hand side.

Similar comparisons are made in Figure 7 for $M_{3}=4$. As expected from Figures 1-3 the predictions from (19) and (20) differ from the exact MHD results only for $M_{A} \lesssim 2-3$ at intermediate $\theta\left(30^{\circ}\right.$ and $\left.60^{\circ}\right)$ but agree very well down to $M_{A}=1.5$ at $\theta=0^{\circ}$ and $90^{\circ}$. Predictions based on the phenomenological model, however, show much larger deviations from the exact MHD solution at $\theta=0^{\circ}$ and $\theta=90^{\circ}$. For the intermediate angles the phenomenological $M_{m s}$ model exhibits more modest deviations from the numerical MHD solutions but still generally show larger differences than the analytic solutions do. As $\theta$ increases toward $90^{\circ}$, the predictions of the phenomenological model are initially larger but eventually smaller than the MHD results for all $M_{A}$. These differences appear suitable for experimental testing.

For the case $\theta=0^{\circ}$, where both the analytic solution and the earlier Zhuang-Russell solution agree precisely with the exact MHD solution, Figures 6 and 7 show that the magnetosonic phenomenological model (replace $M_{s}$ in (22) by $M_{m s}$ ) exhibits a greater than $25 \%$ overshoot of the MHD solution for $\mathrm{X}$ at $M_{A}=2$. Thus the phenomenological model exhibits a dramatic failure at low $\theta$, as was pointed out by Cairns and Grabbe [1994, Figure 5]. Note ironically that the size of this error increases as $M_{s}$ increases. The phenomenological
"Alfven" model (substitute $M_{A}$ in (22) for $M_{B}$ ), which was sometimes compared with data in the 1970 s [e.g., Fairfield, 1971; Formisano et al., 1971], corresponds to the magnetosonic form at $\theta=0^{\circ}$ extended to model all $\theta$. Note again that the predicted $a$, scale is not reliable for any of the models at $\theta=0^{\circ}$ since the empirical relation (21) breaks down there. While combining the MHD expression for $X$ with the empirical relation (21) leads to the MHD models predicting standoff distances that are independent of $M_{A}$, the work by Spreiter and Rizzi [1974] for $\theta=0^{\circ}$ predicts that $a$, actually decreases with decreasing $M_{A}$; the behavior found by Spreiter and Rizzi is exactly opposite to that predicted for the magnetosonic and Alfven phenomenological models.

For the case $\theta=30^{\circ}$, the phenomenological model exhibits a smaller error than at $\theta=0^{\circ}$. It reaches a maximum overshoot of almost $10 \%$ at around $M_{A}=3$ for the $M_{1}=8$ case in Figure 6 , but this maximum falls dramatically as $M_{8}$ decreases in Figure 7 . The analytic solution is much more accurate, and only shows $>1 \%$ deviation as $M_{A}$ becomes close to 2 . For the case $\theta=60^{\circ}$, the phenomenological model exhibits a few percent undershoot. The analytic model is very close to the exact numerical solution for $M_{A}>3$ but exhibits a smaller overshoot at $M_{A}<3$.

For the $\theta=90^{\circ}$ case the analytic solution is equal to the exact solution, but the magnetosonic model exhibits a sizeable error. While the error in the phenomenological model is only a few percent in Figures 6 and 7, there is no error in the analytic model for $\theta=90^{\circ}$ and almost none for $\theta$ close to $90^{\circ}$. Thus even though one can argue that the magnetosonic model is a reasonably good approximation near $\theta=90^{\circ}$, the analytic MHD solution is clearly preferable because it is a much more accurate model and because it is based on MHD plasma theory rather than phenomenology.

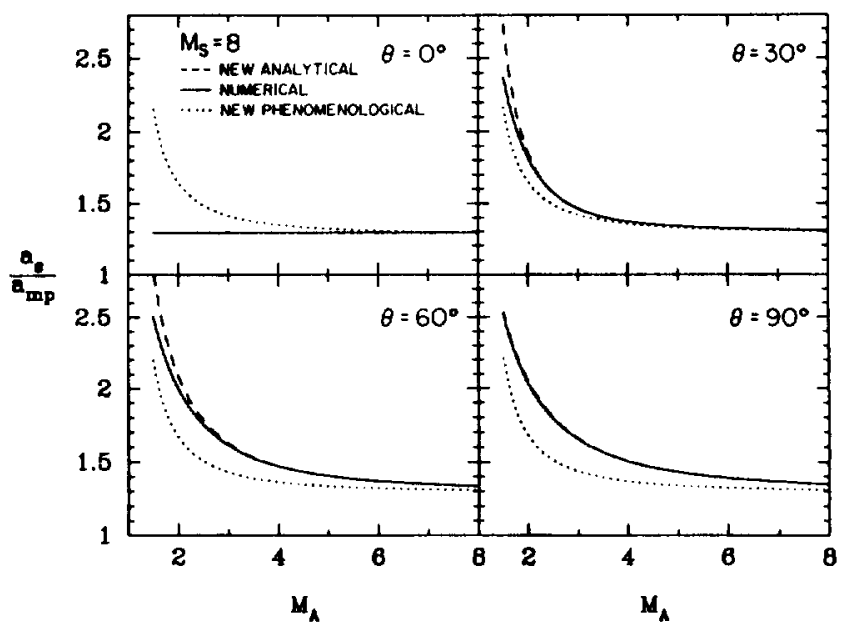

Figure 8. Comparison between the second-order analytical MHD solution (dashed lines), the numerical MHD solution (solid lines) and new phenomenological model of Farris and Russell [1994] (dotted line) using different empirical models. Here $\gamma=5 / 3$ and $M_{s}=8$. The axis on the left-hand side is the predicted ratio $a_{s} / a_{m p}$. 


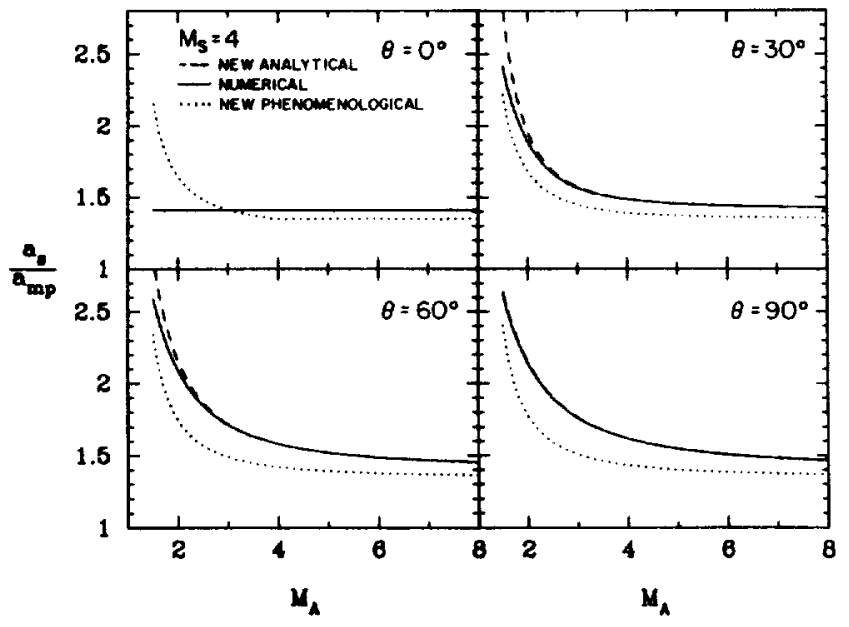

Figure 9. Same as Figure 8 for $M_{\mathrm{s}}=4$.

In Figs. 8 and 9 comparisons of the MHD analytical solutions are made with the new phenomenological model of Farris and Russell [1994] for $\theta=0^{\circ}, 30^{\circ}, 60^{\circ}$, and $90^{\circ}$, for $M_{A}=8$ and $M_{A}=4$, respectively. Once again the analytical and numerical MHD solutions for $X$ are combined with Cairns and Lyon's [1995] empirical relation to predict $a_{3} / a_{m p}$. No modifications are made to the Farris and Russell model since it is intended to account for shock locations at low $M_{A}$ and $M_{m s}$ without change. It is obvious that the two models make very different predictions for all $\theta$ and $M_{A}<10$. These differences are clearly amenable for observational testing. It should be noted that in general the relative differences between the MHD model and the phenomenological model are sizeably larger for $M_{A} \gtrsim 1.5$ at $\theta=30^{\circ}, 60^{\circ}$ and $90^{\circ}$ than they were in Figures 6 and 7 (which have identical $j$ and $k$ in (22)). The predictions of the Farris and Russell model generally undershoots those of the MHD model. The increased difference between Figures 6 and 7 and Figures 8 and 9 indicate a major discrepancy between the predictions of the MHD model and the phenomenological model. Figure 9 indicates that the discrepancy increases in size as $M$ s gets smaller, and that the two models now almost never agree for all $\theta$.

\section{Conclusions}

Perturbation theory was used to obtain two analytical solutions for the density jump ratio $\mathrm{X}$ that satisfy the Rankine-Hugoniot conditions for the Earth's bow shock. These two solutions are accurate for most conditions that exist in the solar wind. By solving the MHD jump equation analytically in a direct perturbation expansion in the Alfven and sonic Mach numbers, the second-order perturbation solution (19) is obtained. The earlier result obtained by Zhang and Russell in a more difficult fashion is the same as the first-order solution of this perturbation expansion. This solution quite accurately matches the exact numerical MHD solution, except when $\theta$ is very close to $90^{\circ}$ while $M_{s}$ or $M_{A}$ is relatively small. For $\theta \sim 90^{\circ}$ the solution (20) much more accurately predicts the value for the density ratio. This second solution is quasi-perpendicular in nature and is generally also of second order in perturbation, of higher order as $\theta$ approaches $90^{\circ}$, and becomes exact at $\theta=90^{\circ}$.

Taken together, (19) and (20) constitute reasonably simple analytical relationships derived from MHD theory for the density jump across the bow shock. When combined with appropriate models at the subsolar region (bow shock nose) for the relation between $X$ and the bow shock's standoff ratio $a_{s} / a_{m p}$, they can be easily used for comparison with data. Two recent empirical models were used for comparisons and definite differences were found in their predictions. Equations (19) and (20) more accurately describe exact theoretical MHD results than the phenomenological models for $X$ that have been used (in conjunction with the SeiffSpreiter empirical model) for comparison with spacecraft data for many years. Those two equations, when combined with the most appropriate empirical model, constitute decidedly better replacements to use for that purpose.

A goal for future research in this area is to use MHD theory to develop a fully analytic model for the relationship between $\mathrm{X}$ and $a_{s} / a_{m p}$ to replace the empirical models. Only when that is accomplished will a completely theoretical MHD model be available to predict the bow shock's location. Developing a full MHD description for the solar wind flow at the Earth's bow shock, which will require a self-consistent threedimensional determination of the shock location, shape, and jump conditions as a function of $M_{A}, M_{s}, \gamma$, and $\theta$, should then be possible.

Acknowledgments. This research was supported in part by NSF grant ATM-9312263 and NASA grant NAGW2040.

The Editor thanks G. L. Siscoe and J. A. Slavin for their assistance in evaluating this paper.

\section{References}

Cairns, I.H., and C.L. Grabbe, Towards an MHD Theory for the Standoff distance of Earth's bow shock, Geophys. Res. Lett., 21, 2781, 1994.

Cairns, I.H., and J.G. Lyon, MHD simulations of Earth's bow shock at low Mach numbers: Standoft distances, J. Geophys. Res., in press, 1995.

Cairns, I.H., D.H. Fairfield, R.R. Anderson, V.E.H. Carlton, K.I. Paularena, and A.J. Lazarus, Unusual locations of Earth's bow shock on 24-25 September 1987: Mach number effects, J. Geophys. Res., 100, 47, 1995.

Fairfield, D.H., Average and unusual locations of the Earth's magnetopause and bow shock, J. Geophys. Res., 76, 6700, 1971.

Farris, M. H., and C. T. Russell, Determining the standoff 
distance of the bow shock: Mach number dependence and use of models, J. Geophys. Res., 99, 17,681, 1994.

Farris, M.H., S.M. Petrinec, and C.T. Russell, The thickness of the magnetosheath: Constraints on the polytropic index, Geophys. Res. Lett., 18, 1821, 1991.

Formisano, V., P.C. Hedgecock, G. Moreno, J. Sear, and D. Bollea, Observations of Earth's bow shock for low mach numbers, Planet. Space Sci., 19, 1519, 1971.

Holzer, R.E., and J.A. Slavin, Magnetic flux transfer associated with expansions and contractions of the dayside magnetosphere, J. Geophys. Res., 83, 3831, 1978.

Nayfeh, A., Introduction to Perturbation Techniques, John Wiley, New York, 1993.

Peredo, M., J.A. Slavin, E. Mazur, and S.A. Curtis, Threedimensional position and shape of the bow shock and their variation with Alfvenic, sonic, and magnetosonic Mach number and interplanetary magnetic field orientation, $J$. Geophys. Res., 100, 7907, 1995.

Russell, C.T., Planetary bow shocks, in Collisionless Shocks in the Heliosphere: Reviews, of Current Research, Geophys. Monogr. Ser., vol. 35, edited by B.T. Tsurutani and R.G. Stone, p. 109, AGU, Washington, D.C., 1985.

Russell, C.T., and T.-L. Zhang, Unusually distant bow shock encounters at Venus, Geophys. Res. Lett., 19, 833, 1992.

Seiff A., Gasdynamics in space exploration, NASA Spec. Publ., 24, 1962.

Sibeck, D.G., R.E. Lopez, and E.C. Roelof, Solar wind control of the magnetopause shape, location, and motion, $J$. Geophys. Res., 96, 5489, 1991.

Slavin, J.A., R.E. Holzer, J.R. Spreiter, S.S. Stahara, and
D.S. Chausee, Solar wind flow about the terrestrial planets, 2, Comparisons with gas dynamic theory and implications for solar-planetary relations, J. Geophys. Res., 88, $19,1983$.

Spreiter, J.R., and A.W. Rizzi, Aligned magnetohydrodynamic solution for solar wind flow past the Earth's magnetosphere, Acta Astronaut., 1, 15, 1974.

Spreiter, J.R., and S.S. Stahara, A new predictive model for determining solar wind-terrestrial planet interactions, $J$. Geophys. Res., 85, 6769, 1980.

Spreiter, J.R., A.L. Summers, and A.Y. Alksne, Hydromagnetic flow around the magnetosphere, Planet. Space Sci., 14, 223, 1966.

Tidman, D.A., and N.A. Krall, Shock Waves in Collisionless Plasmas, John Wiley, New York, 1971.

Winterhalter, D., M.G. Kivelson, R.J. Walker, and C.T. Russell, Magnetic field change across the Earth's bow shock: Comparison between observations and theory, $J$. Geophys. Res., 90, 3925, 1985.

Zhuang, H.C., and C.T. Russell, An analytic treatment of the structure of the bow shock and magnetosheath, $J$. Geophys. Res., 86, 2191, 1981.

I.H. Cairns and C.L.Grabbe, Department of Physics and Astronomy, University of Iowa, Iowa City, IA 522421479. (e-mail: grabbe@iowa.physics .uiowa.edu; ihc@space. physics.uiowa.edu)

(Received January 31, 1995; revised April 5, 1995; accepted April 18, 1995.) 
